\title{
Diagnóstico pré-natal de peritonite meconial - relato de caso
}

\section{Prenatal diagnosis of meconium peritonitis - case report}

\author{
Márden Pinheiro Teixeira Costa ${ }^{1}$. Helvécio Neves Feitosa ${ }^{2}$. Kárita de Melo Cordeiro ${ }^{3}$. João Henrique Freitas \\ Colares ${ }^{4}$. Francisco das Chagas Brilhante Barros ${ }^{4}$. Sergio Orceliano Moreira ${ }^{4}$. Francisco Herlânio Costa \\ Carvalho ${ }^{2}$.
}

1 Médico residente de Ginecologia/Obstetrícia da Maternidade Escola Assis Chateaubriand (MEAC), Universidade Federal do Ceará (UFC), Fortaleza, Ceará, Brasil. 2 Doutorado em Medicina (Obstetrícia), Professor Adjunto do Departamento de Saúde Materno Infantil da Universidade Federal do Ceará (UFC), Fortaleza, Ceará, Brasil. 3 Médica residente de Medicina Fetal da Maternidade Escola Assis Chateaubriand (MEAC), Universidade Federal do Ceará (UFC), Fortaleza, Ceará, Brasil. 4 Cirurgião pediátrico da Maternidade Escola Assis Chateaubriand (MEAC).

\section{RESUMO}

Introdução: a peritonite meconial deve ser considerada no diagnóstico diferencial em pacientes que desenvolvem grandes cistos abdominais. Avanços nas técnicas de ultrassonografia permitem reconhecer mais precocemente os sinais pré-natais indicativos da patologia e ajudam na redução das taxas de morbimortalidade. Relato de caso: A.E.S.S., 21 anos, primigesta, realizou ultrassonografia obstétrica no dia 24/03/15, sendo evidenciado imagem anecóica, intra-abdominal sugestiva de íleo meconial. Na $39^{\text {a }}$ semana foi submetida a parto abdominal devido a macrossomia fetal. Recém-nascido (RN), vivo, sexo feminino, escore de Apgar de 3 e 7 no $1^{\circ}$ e $5^{\circ}$ minutos, respectivamente. Evoluiu nos 5 primeiros dias de vida com resíduo gástrico fecalóide, e após avaliação da cirurgia pediátrica foi indicada laparotomia exploradora pela hipótese de íleo meconial. Os achados foram atresia de íleo terminal com perfuração e bloqueio de alças, sendo realizado ressecção dos segmentos atrésicos e anastomose término-terminal. Necessitou de nova abordagem cirúrgica e progrediu com quadro séptico. No primeiro dia de pós-operatório evoluiu para óbito. Conclusão: no presente caso, apesar da visualização das alterações ultrassonográficas no pré-natal, o diagnóstico definitivo só foi possível após abordagem cirúrgica. Caso fosse diagnosticado precocemente, a evolução neonatal poderia ter sido satisfatória, com antecipação das cirurgias, mesmo com os altos índices de mortalidades.

Palavras-chave: Peritonite. Mecônio. Ultrassonografia. Cuidado pré-natal.

\section{ABSTRACT}

Introduction: meconium peritonitis should be considered in the differential diagnosis in patients who develop large abdominal cysts. Advance of ultrasonography techniques for recognizing earlier indicative prenatal signs of disease and helps in reducing morbidity and mortality rates. Case report: A.E.S.S., 21 years old, primigravidae. Obstetric ultrasonography performed on 24/03/15, being evidenced anechoic image, elongated, suggesting intra-abdominal meconial ileum. In the 39th week was subjected to abdominal childbirth due to fetal macrosomia. Newborn, live, female, Apgar score at 3 and 7 at 1 and 5 minutes, respectively. It evolved within the first 5 days of life with fecaloid gastric residuals daily, and after evaluation of pediatric surgery was indicated explorative laparotomy for meconium ileus hypothesis. The findings were atresia of the terminal ileum with perforation and lock handles, being performed resection of the atresia and end-to-end anastomosis segments. He needed another surgical approach and progressed to sepsis. On the first day after surgery he died. Conclusion: in this case, although the visualization of ultrasonography changes in prenatal the definitive diagnosis was only possible after surgical approach. Case was diagnosed early, the neonatal outcome could have been satisfactory, with anticipation of surgery, even with high rates of mortality.

Keywords: Peritonitis. Meconium. Ultrasonography. Prenatal care.

Autor correspondente: Márden Pinheiro Teixeira Costa, Rua Leonardo Mota, 1200, apartamento 503, Aldeota, Fortaleza, Ceará. CEP: .Telefone: +55 85 99772-0591. E-mail: mardenptc@gmail.com

Conflito de interesses: Não há qualquer conflito de interesses por parte de qualquer um dos autores.

Recebido em: 19 Jan 2016; Revisado em: 09 Mar 2016; Aceito em: 14 Mar 2016. 


\section{INTRODUÇÃO}

Peritonite meconial é uma peritonite química estéril causada por perfuração intestinal com extravasamento de mecônio intrauterino, e deve ser considerada no diagnóstico diferencial em pacientes que desenvolvem grandes cistos abdominais com ar e conteúdo fluido. ${ }^{1,2}$ Esta patologia é de ocorrência rara, sendo geralmente estimada em 1 por 30.000 a 35.000 nascimentos. ${ }^{2,3}$

Em sua etiopatogênese estão envolvidos diversos fatores, como a obstrução orgânica (volvo, atresia ou íleo meconial), patologia vascular e funcional ou etiologia viral (hepatite, varicela, citomegalovírus, parvovírus). ${ }^{1,4}$

As malformações pré-natais do trato gastrintestinal estão sendo reconhecidas com maior frequência. Isto ocorre em parte devido à melhora do avanço tecnológico na imagem da ultrassonografia, bem como pela melhor compreensão através do ultrassom das manifestações gastrointestinais. Com frequência o sinal ultrassonográfico inicial é um sinal indireto, mais do que a própria anomalia. ${ }^{5}$

O diagnóstico pré-natal de peritonite meconial tem sido mais comum nos últimos anos, devido à presença de achados ultrassonográficos de polidrâmnio, ascite fetal, dilatação de alças intestinais e calcificações. O diagnóstico também deve ser suspeitado em feto que apresenta imagem de obstrução intestinal e desenvolve ascite e hidropisia. ${ }^{6,2}$

Neste estudo, os autores relatam um caso de cisto abdominal resultado de peritonite meconial suspeitada antes e com diagnóstico confirmado após o nascimento. $\mathrm{O}$ caso em questão tem relevância não apenas pela raridade, mas também enfatizando a importância da ultrassonografia e outros exames de imagem no pré e pós-natal, na tentativa de se diagnosticar mais precocemente e atuar na redução das taxas de morbimortalidade.

\section{RELATO DO CASO}

A.E.S.S., 21 anos, primigesta, foi encaminhada ao pré-natal de alto risco do serviço de medicina materno-fetal da Maternidade Escola Assis Chateaubriand (MEAC) em 24/03/15 com idade gestacional de 27 semanas e 2 dias.
Trazia ultrassonografia realizada com idade gestacional de 22 semanas, visualizando distensão de segmentos de alças intestinais de localização periférica, sendo interrogado megacólon congênito. Sem queixas clínicas na primeira consulta. Negava contato com doenças infecciosas, uso de álcool e/ou drogas, exposição à radiação, manobras abortivas e quaisquer outras intercorrências. Negava antecedentes familiares de malformações.

Foi realizado ecocardiograma fetal sem anormalidades. Foi oferecido amniocentese para estudo do cariótipo, porém a paciente recusou.

Foi realizada ultrassonografia no serviço de medicina fetal da MEAC, sendo evidenciado intestino hiperecogênico, imagem anecóica, alongada, intra-abdominal medindo $2,3 \times 0,5 \mathrm{~cm}$ e dilatação de alças intestinais, interrogado hipótese de íleo meconial.

Foi submetida à ressonância nuclear magnética fetal, sendo visualizada formação cística intra-peritoneal, descrito como imagem inespecífica. Fígado, baço e rins normais. Líquido livre em cavidade abdominal em pequena quantidade. Dentre as possibilidades diagnósticas, considerou-se a hipótese de hamartoma mesenquimal exofítico do fígado, considerando os diferenciais de lesão cística primária da cavidade (linfangioma ou cisto de duplicação entérico). Mais remotamente, levantada a possibilidade de cistos ovarianos com extensão abdominal.

A paciente foi submetida a mais duas ultrassonografias, a primeira constatando alças intestinais dilatadas, massa hiperecogênica de parede intra-abdominal, sub-hepática à direita, medindo 4,4 x 2,6 cm (Figura 1), além de índice de líquido amniótico (ILA) de $292 \mathrm{~mm}$. Interrogou-se, então, obstrução de alça intestinal, ileal, jejunal? Íleo meconial? Ânus imperfurado? Doença de Hirschsprung?

$\mathrm{Na}$ segunda ultrassonografia se manteve a imagem da massa intra-abdominal hiperecogênica, com forte sombra acústica posterior, medindo 19 × 35 × $20 \mathrm{~mm}$ e volume de $7,3 \mathrm{~cm}^{3}$ em região anterior à direita da veia umbilical, em seu trajeto intra-abdominal. Notava-se ainda, dilatação de alças intestinais, com diâmetro luminal de 2,0 cm e ILA de $154 \mathrm{~mm}$.

Figura 1. Ultassonografia fetal evidenciando dilatação de alças intestinais e massa hiperecogênica sub-hepática.

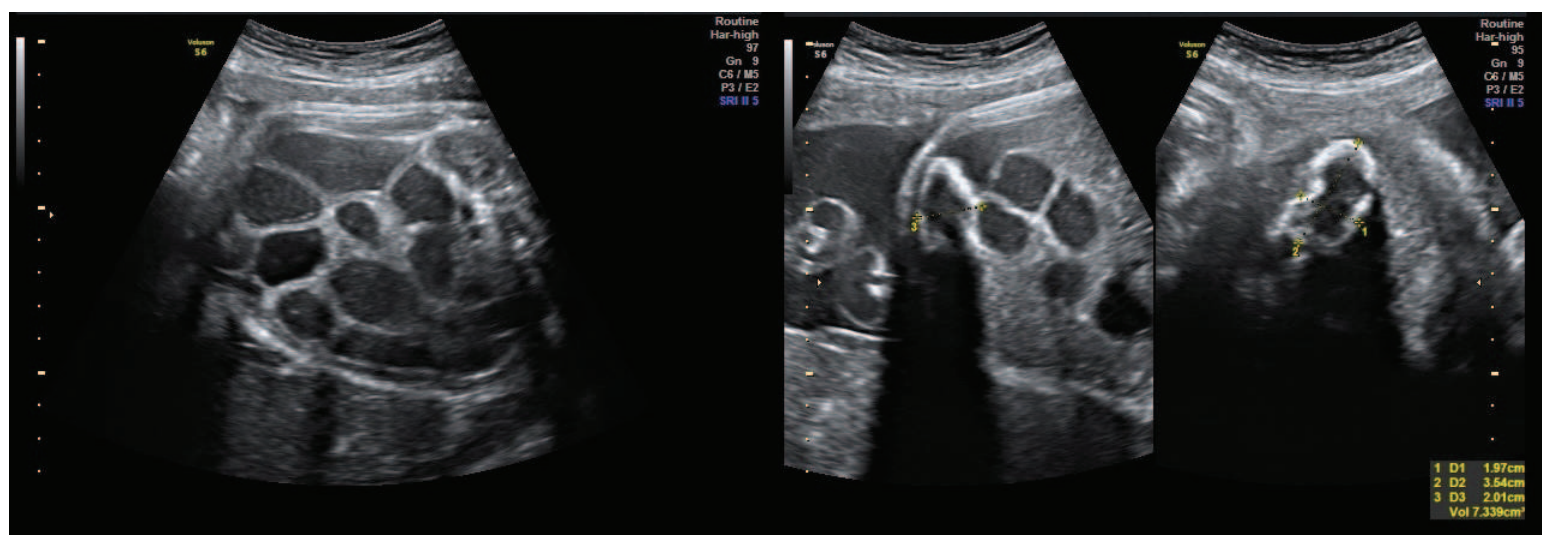


$\mathrm{Na} 39^{\mathrm{a}}$ semana foi submetida a parto abdominal devido macrossomia fetal (peso estimado de $4.692 \mathrm{~g}$ ). Recém-nascido (RN), vivo, sexo feminino, escore de Apgar de 3 e 7 no $1^{\circ}$ e $5^{\circ}$ minutos, respectivamente. Foi imediatamente intubado com tubo orotraqueal tamanho 3,5 e encaminhado à Unidade de Terapia Intensiva Neonatal (UTIN). Ao exame físico após nascimento foi evidenciado gemência, cianose e o abdome encontrava-se globoso, tenso, com presença de massa em hemiabdome à direita.

Em menos de 24 horas foi extubado, colocado em HOOD com FIO $30 \%$ e sendo constatado resíduo gástrico bilioso de $146 \mathrm{ml}$ em 24 horas. Foi avaliado pela cirurgia pediátrica que solicitou Raio-X e ultrassonografia abdominais, sendo visualizado no primeiro distensão de alças e níveis hidroaéreos (Figura 2) e no segundo exame volumosas imagens tubulares anecóicas, tortuosas, com conteúdo líquido, exibindo peristalse em andar inferior do abdome; fígado, baço e rins normais.

Figura 2. Raio-X de abdome, anterior-posterior e perfil, evidenciando distensão de alças intestinais.

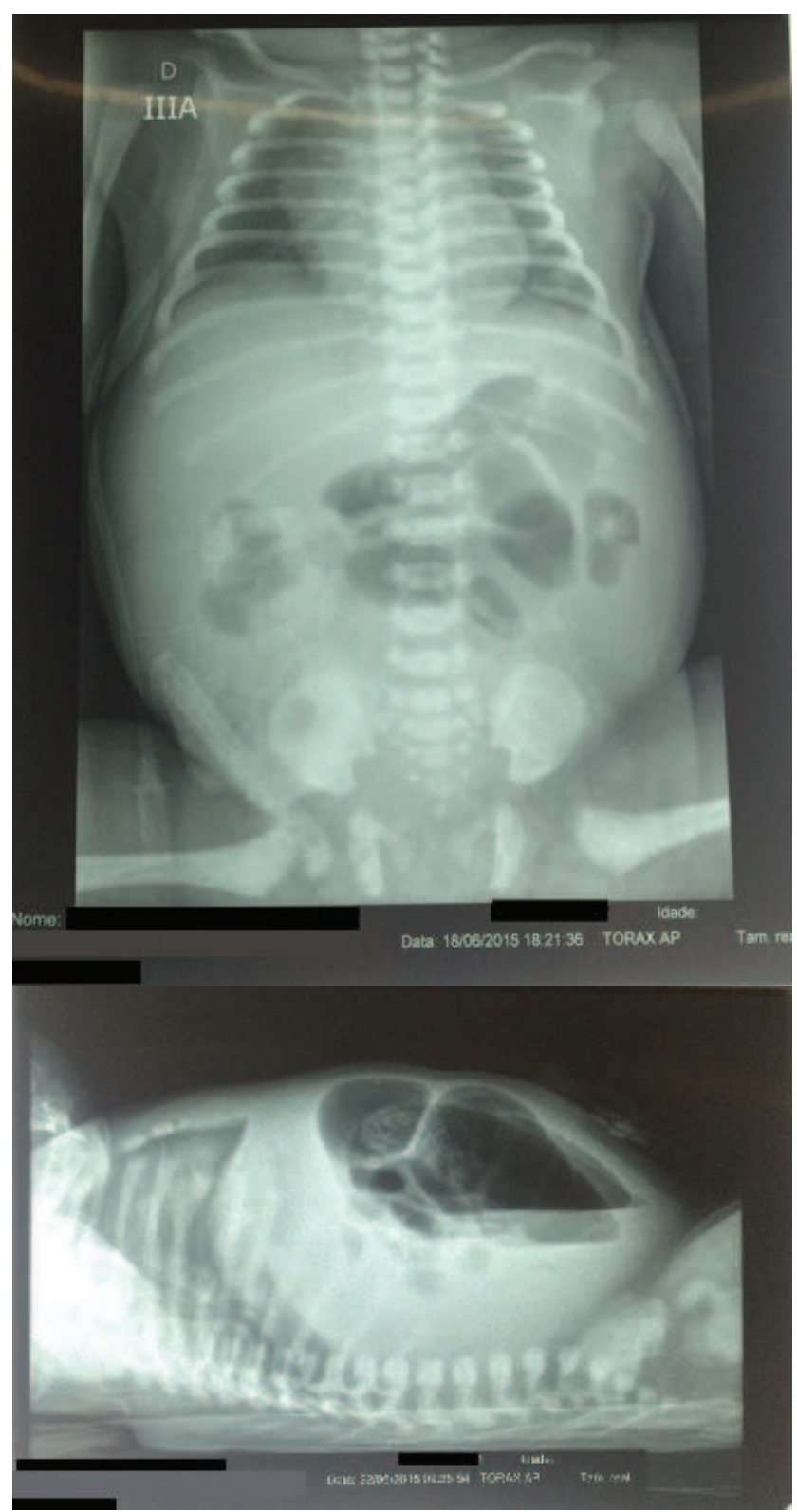

Chegou a receber dieta via oral e evoluiu com melhora do desconforto respiratório, com suspensão de oxigenioterapia, porém persistia com distensão abdominal com ruídos hidroaéreo (RHA) diminuídos e oligúria. RN evoluiu nos 5 primeiros dias de vida com resíduo gástrico bilioso diariamente, evoluindo para fecalóide. Hemograma com neutrofilia.

Após nova avaliação da cirurgia pediátrica foi indicada laparotomia exploradora. Os seguintes achados foram descritos: atresia de íleo terminal com provável perfuração e bloqueio de alças, comprometendo ceco e cólon ascendente, além de múltiplas aderências. Realizada ressecção dos segmentos atrésicos extendendo-se $10 \mathrm{~cm}$ dos segmentos e anastomose término-terminal. Iniciado profilaticamente esquema antibiótico com penicilina cristalina e gentamicina, além da necessidade de intubação orotraqueal e dieta zero associada a nutrição parenteral. No dia seguinte foi acrescentado tazocin profilático por 3 dias.

No $5^{\circ}$ dia pós operatório o RN evoluiu com taquidispnéia, taquicardia, resíduo gástrico bilioso, porém com evacuações presentes. Hemograma encontrava-se alterado sugerindo sepse. Foi então iniciado antibioticoterapia com cefepime e vancomicina.

O resultado da hemocultura detectou infecção por Pseudomonas aeruginosa. Evoluiu com bradicardia e nova intubação orotraqueal.

Apresentou piora clínica, sendo necessário fazer uso de óxido nítrico (acidose respiratória), necessitando de drogas vasoativas, sendo então iniciado dopamina e dobutamina.

Houve surgimento de rash máculo-papular disseminado com hiperemia de parede abdominal, suspeitando-se de deiscência e abscesso abdominal. Avaliado pela cirurgia pediátrica, foi submetido a drenagem de cavidade abdominal, após confirmação ultrassonográfica, com extravasamento de material violáceo, fezes e ar. Nova laparotomia evidenciou presença de fezes em cavidade peritoneal, aderências firmes entre alças e deiscência parcial de anastomose íleo-cólica. Realizada ileostomia com lise de aderências. No primeiro dia de pós-operatório apresentou hipotensão mesmo em uso de drogas vasoativas; no entanto, evoluiu para óbito.

\section{DISCUSSÃO}

No pré-natal vários achados ultrassonográficos incluindo dilatação intestinal, ascite e polidrâmnio podem estar associados com peritonite meconial. ${ }^{7,8}$ No entanto, a presença de calcificação é o mais importante e geralmente está associada ao diagnóstico.

Peritonite meconial diagnosticada no pré-natal difere daquela diagnosticada em recém-nascidos. A tendência é que as formas menos severas sejam diagnosticadas antes do nascimento, e o ultrassom seja mais sensível na detecção de calcificações. Muitos dos casos diagnosticados ainda intrauterinos podem permanecer assintomáticos mesmo após o parto, e aquela 
diagnosticada em recém-nascidos representa a forma mais grave da doença com associação de obstrução intestinal e peritonite. Portanto, o prognóstico da patologia diagnosticada no pré-natal é obviamente melhor do que diagnosticado no neonato. ${ }^{7}$

No caso apresentado, apesar da visualização de alterações ultrassonográficas no pré-natal, como dilatação de alças intestinais, massa cística intra-peritoneal e polidrâmnio, o diagnóstico definitivo de atresia de íleo terminal só foi possível após abordagem cirúrgica já no $7^{\circ}$ dia de vida. Não foram evidenciados outros sinais sugestivos descritos na literatura como calcificações císticas e ascite, dificultando ainda mais o diagnóstico ultrassonográfico no pré-natal, incluindo nosso estudo entre a maioria dos casos suspeitos de peritonite meconial, onde menos de dez casos descritos tiveram o correto diagnóstico intrauterino. ${ }^{9,10}$

\section{REFERÊNCIAS}

1. Dirkes K, Crombleholme TM, Craigo SD, Latchaw LA, Jacir $\mathrm{NN}$, Harris $\mathrm{BH}$, et al. The natural history of meconium peritonitis diagnosed in utero. J Pediatr Surg. 1995;30(7):979-82.

2. Amagada JO, Premkumar G, Arnold JM, Davies C. Prenatal meconium peritonitis managed expectantly. J Obstet Gynaecol. 2004;24(3):311-2.

3. Brisighelli A Neto, Caetano SM, Sorrenti R. Ascite fetal isolada. Rev Cienc Med Campinas. 2007;16(1):57-60.

4. Hyett J. Intra-abdominal masses: prenatal differential diagnosis and management. Prenat Diagn. 2008;28(7):645-55.

5. Saleh N, Geipel A, Gembruch U, Heep A, Heydweiller A, Bartmann P, et al. Prenatal diagnosis and postnatal management of meconium peritonitis. J Perinat Med. 2009;37(5):535-8.

6. Konje JC, Chazal R, MacFadyen U, Taylor DJ. Antenatal diagnosis
Convém ressaltar que, apesar dos avanços diagnósticos pré-natais com a ressonância magnética, a ultrassonografia ainda se mantém como o exame de melhor avaliação para rastreamento e diagnóstico de malformações fetais; além de mais facilmente acessível.

Concordamos que a possibilidade de não confiança no diagnóstico pré-natal, associado a evolução inicialmente favorável, pode ter complicado a evolução neonatal no presente caso, uma vez que houve retardo no diagnóstico pósnatal, tendo sido realizada intervenção cirúrgica tardiamente, com o recém-nascido em condições clínicas menos estáveis. $\mathrm{O}$ óbito, no $18^{\circ}$ dia pós-operatório associou-se com o quadro de sepse, talvez evitável se a cirurgia tivesse sido indicada precocemente. Importante destacar que a mortalidade pré-natal ainda é elevada na presença de malformações intestinais, apesar do diagnóstico precoce intrauterino.

and management of meconium peritonitis: a case report and review of the literature. Ultrasound Obstet Gynecol. 1995;6(1):66-9.

7. Ramesh JC, Chow TW, Yik YI, Ramanujam TM. Meconium peritonitis: prenatal diagnosis and postnatal management - a case report. Med J Malaysia. 1999;54(4):528-30.

8. Minato M, Okada T, Miyagi H, Honda S, Takazawa K, Kubota $\mathrm{KC}$, et al. Meconium pseudocyst with particular pathologic findings: a case report and review of the literature. J Pediatr Surg. 2012;47(4):e9-12.

9. Yang WT, Ho SS, Metreweli C. Case report: antenatal sonographic diagnosis of meconium peritonitis and subsequent evolving meconium pseudocyst formation without peritoneal calcification. Clin Radiol. 1997;52(6):477-9.

10. Agboola AJ, Stokes IM, Jones A. An unusual presentation of meconium peritonitis. J Obstet Gynaecol. 2004;24(1):90-1

\section{Como citar:}

Costa MP, Feitosa HN, Cordeiro KM, Colares JH, Barros FC, Moreira SO, et al. Diagnóstico pré-natal de peritonite meconial - relato de caso. Rev Med UFC. 2017 jan-abr;57(1):52-55. 